\title{
Could clinical experience during clerkship enhance students' clinical performance?
}

\author{
Ji Young Kim and Sun Jung Myung*
}

\begin{abstract}
Background: Medical students learn and practice various clinical skills during clinical clerkship. Patient encounters are important for developing clinical thinking, communication skills, and professional attitude. We investigated whether the amount of clinical experience during clerkship correlated with students' clinical competency and students' perception of effectiveness of their clerkship on it.

Methods: Fourth year medical students undertook the Objective Structured Clinical Examinations (OSCE) in August 2012. Students provided the number of patients for whom they took medical histories or performed physical examinations during clerkship and provided feedback as to whether or not the clinical clerkship was helpful in preparing OSCE. The correlation between the OSCE score and number of patients was analyzed.

Results: One hundred thirty students completed the questionnaire (86.6\%). OSCE scores correlated with the total number of patients encountered for physical examinations (correlation coefficient, $0.274 ; p=0.0105$ ). Cumulative 3-year GPAs were positively correlated with OSCE scores (correlation coefficient, 0.330; $p=0.0001$ ). Most (92.3\%) answered that their clinical clerkship was helpful in preparing them for the OSCE; however, only 20\% felt that their clinical clerkship was most helpful. Others felt that role playing (38.46\%) or the guide book (33.84\%) was most helpful.

Conclusions: The amount of clinical experience during the students' clerkship had a small but positive relationship with students' clinical performance. Further research to elucidate the influence of clinical experience on clinical competency is needed.
\end{abstract}

Keywords: Objective structured clinical examination, Clinical clerkship, Medical education, Clinical competency

\section{Background}

Traditionally, we believe that clinical competence is developed from clinical experience. Learning clinical skills is a time-consuming and gradual process, and clerkship is the time in which students must learn and practice various clinical skills. During clinical clerkship, students encounter patients in the ward or at outpatient clinics, and these encounters are at the core of the program for developing students' clinical competency [1,2].

Nowadays, early clinical exposure is warranted by experts in medical education [3-6], and we encourage students to actively participate in clinical practice. We believe that these experiences will enhance the students' clinical competency. However, clinical experience during

\footnotetext{
* Correspondence: issac73@snu.ac.kr

Office of Medical Education, Seoul National University College of Medicine, 103 Daehak-ro, Jongno-gu, Seoul 110-799, Republic of Korea
}

clinical clerkship varies among students, and the relationship between students' clinical experience and their subsequent performance has always been recognized as complex [7].

Although much effort has been made to find correlations between the number or variety of patients seen during clerkships and clinical performance at the end of the clerkship, evidence regarding the educational value of patient encounters is still limited. The nature of the examinations given, such as the written knowledgebased examinations and the variety of quality of clinical experiences, were reasons for the observed lack of correlations [8-10]. Additionally, the quality of the clinical supervisor was important for developing clinical competence $[11,12]$. Although the influence of clerkship experiences on clinical competence yielded contradictory results, medical students evaluated the exposure to real 
patients as very important [10]. Real-life contextual experience by clinical exposure added value in acquiring clinical competence, whereas paper-based case tutorials also provided students with clinical knowledge [13].

Because it is important that we understand how students benefit from clinical experience and what types of experience are most beneficial, we tried to determine whether the amount of clinical experience during clerkship in each department correlates with the clinical competency of the students and evaluated medical students' perceptions of the effectiveness of their clerkships on their clinical performance examination.

\section{Methods}

The current undergraduate curriculum of Seoul National University College of Medicine (SNUCM; Seoul, South Korea) includes two years of preclinical study followed by two years of clinical study. The third year course is run by the departments of internal medicine, surgery, pediatrics, psychiatry, obstetrics and gynecology etc. The fourth year course is composed of elective clerkships during which each student can choose his or her own clerkship schedule. During clinical clerkship, students learn key components of clinical practice by encountering patients, practicing clinical skills, and attending tutoring sessions. The students are required to maintain a logbook that lists of their experiences with patients and exposure to core problems in each department.

We used the Objective Structured Clinical Examination (OSCE) as a tool for assessing clinical performance because measuring medical students' clinical competency using OSCEs has become increasingly widespread, and evidence of the validity of the test is mounting [14-17].

In August 2012, all fourth-year medical students at SNUCM participated in the OSCE, which consists of 8 stations presenting the following 8 clinical cases: syncope, abdominal pain, palpitation, alcohol abuse problems, hemoptysis, polyuria, and constipation. The OSCE was developed by the Seoul Kyeonggi CPX Consortium. Each case required 16 minutes to administer, with $1 \mathrm{~min}$ to introduce the next case, $10 \mathrm{~min}$ for the studentstandardized patient encounter, and $5 \mathrm{~min}$ after the encounter for the student to report the most probable diagnosis. The student's performance was evaluated by trained standardized patients (SPs) using a checklist $[18,19]$ (see Additional file 1 for the format of the scoring rubric of OSCE), and major evaluation components are overall assessment, history taking, physical examination, physician's manner, patient education, and physicianpatient interaction (PPI). Evaluating performance by trained SPs using checklist is regarded as reliable methods and this has been verified by many previous reports $[16,18,19]$. At the end of the exam, the students were asked to complete a questionnaire inquiring about the number of patients for whom they took a medical history and physical examination based on the logbook that each student kept during their clerkship. The questionnaire also has items for students' perceptions of the effectiveness of the clerkship on their clinical performance examination (see Additional file 2 for items).

Grade point average (GPA), OSCE scores from the encounters with standardized patients (including history taking, physical examination, and patient-physician interaction), and the number of patients encountered during clerkship (especially those for whom students took a history or conducted a physical examination) were obtained and a correlation analysis was done. Statistical analyses were performed using SPSS software (version 19.0; SPSS Inc., Chicago, IL, USA). A $p$-value $<0.05$ was considered statistically significant. The institutional review board provided study approval.

\section{Results}

Number of patients encountered by medical students during clinical clerkship

A total of 150 fourth-year students underwent the OSCE examinations in August 2012. Among them, 130 students (86.6\%) completed the questionnaire. The number of patients that students examined during their clinical clerkship varied widely by department and individual student, ranging from 0 to 30 . The total number of patients encountered during clerkship per year ranged from 5 to 85 (Table 1).

Correlation between the number of patients who medical students encountered during clerkship and their OSCE score

As shown in Table 2, the total OSCE score was statistically significantly correlated with the total number of patients the students conducted a physical examination on during their third year clinical clerkship. OSCE scores for history taking, physical examination, or PPI partly

Table 1 Average number of patients encounter during students' clerkship

\begin{tabular}{llllllllll}
\hline & & IM & GS & OBGY & Ped & Psy & OS & EM & Total \\
\hline History Taking & Min & 2 & 0 & 0 & 1 & 1 & 0 & 0 & 5 \\
& Max & 30 & 30 & 10 & 16 & 20 & 10 & 10 & 85 \\
& Mean & 13.9 & 8.6 & 3.1 & 4.9 & 5.7 & 1.9 & 2.6 & 40.5 \\
& SD & 5.5 & 5.5 & 2.7 & 2.7 & 3.0 & 2.0 & 1.7 & 14.9 \\
Physical Exam & Min & 0 & 0 & 0 & 0 & 0 & 0 & 0 & 0 \\
& Max & 30 & 20 & 10 & 16 & 12 & 20 & 10 & 85 \\
& Mean & 12.9 & 7.9 & 2.5 & 4.5 & 3.6 & 1.7 & 2.3 & 35.4 \\
& SD & 5.4 & 5.2 & 2.6 & 2.7 & 3.4 & 2.3 & 1.6 & 14.3 \\
\hline
\end{tabular}

IM, internal medicine; GS, surgery; OBGY, obstetrics and gynecology; Ped, pediatrics; Psy, psychiatry; OS, orthopedics; EM, emergency medicine. Min, minimum; Max, maximum; SD, standard deviation. 
Table 2 Pearson correlations between the number of patients encountered during clerkship and OSCE score, GPA

\begin{tabular}{|c|c|c|c|c|c|}
\hline & & History taking & Physical examination & PPI & Total score \\
\hline IM (Hx) & $r$ & 0.053 & 0.016 & 0.142 & 0.090 \\
\hline IM (P/E) & $r$ & 0.122 & 0.090 & 0.189 & 0.148 \\
\hline GS $(H x)$ & $r$ & 0.194 & 0.230 & 0.190 & 0.224 \\
\hline GS (P/E) & $r$ & 0.249 & 0.242 & 0.269 & 0.278 \\
\hline $\mathrm{OBGY}(\mathrm{Hx})$ & $r$ & 0.125 & 0.013 & 0.178 & 0.133 \\
\hline OBGY (P/E) & $r$ & 0.083 & -0.030 & 0.161 & 0.102 \\
\hline Ped $(H x)$ & $r$ & 0.050 & 0.037 & 0.164 & 0.089 \\
\hline Ped (P/E) & $r$ & 0.085 & 0.076 & 0.195 & 0.132 \\
\hline Psychiatry (Hx) & $r$ & 0.033 & 0.049 & 0.109 & 0.071 \\
\hline Psychiatry (P/E) & r & 0.147 & 0.172 & 0.308 & 0.245 \\
\hline OS $(H x)$ & r & 0.105 & -0.006 & 0.081 & 0.072 \\
\hline OS (P/E) & r & 0.074 & -0.118 & 0.041 & -0.003 \\
\hline EM $(H x)$ & $r$ & 0.120 & 0.105 & 0.194 & 0.166 \\
\hline EM (P/E) & r & 0.116 & 0.073 & 0.202 & 0.156 \\
\hline \multirow[t]{2}{*}{ Total $(\mathrm{Hx})$} & $r$ & 0.156 & 0.119 & $0.238^{*}$ & 0.198 \\
\hline & $\mathrm{p}$ & 0.148 & 0.174 & 0.036 & 0.095 \\
\hline \multirow[t]{2}{*}{ Total (P/E) } & $r$ & $0.227^{*}$ & 0.160 & $0.336^{*}$ & $0.274^{*}$ \\
\hline & $p$ & 0.045 & 0.148 & $<.0001$ & 0.0105 \\
\hline \multirow[t]{2}{*}{ GPA } & $r$ & $0.271^{*}$ & $0.314^{*}$ & $0.244^{*}$ & $0.330^{*}$ \\
\hline & $p$ & 0.001 & 0.0003 & 0.005 & 0.0001 \\
\hline
\end{tabular}

IM, internal medicine; GS, surgery; OBGY, obstetrics and gynecology; Ped, pediatrics; OS, orthopedics; EM, emergency medicine; Hx, history taking; P/E, physical examination; PPI, physician-patient interaction.

$r=$ correlation coefficient.

$\mathrm{p}=$ adjusted $p$-value by Hochberg's step up method.

${ }^{*} \mathrm{~A} p$-value $<.05$ was considered statistically significant.

correlated with the number of patients encountered during internal medicine, surgery, pediatrics, and psychiatry clerkships. GPA was also significantly correlated with OSCE scores for total, history taking, physical examination, and PPI (Table 2).

\section{Was clinical clerkship helpful for enhancing the clinical competency of students?}

Most students answered that their clinical clerkship was helpful and enhanced their OSCE scores (92.3\% of students scored over 3 on a Likert scale score of 5). However, students also replied that role playing with friends (38.46\%) or reading books dealing with OSCE (33.84\%) were most helpful when preparing for the OSCE rather than clinical clerkship.

\section{Discussion}

In this study, we show that the amount of clinical experience during clerkship has a small but positive relationship with students' clinical performance. In the belief that seeing more patients and encountering more core problems will result in better clinical performance of the students, academic medical school managers try to provide students with more clinical encounters with patients earlier in their medical school training. Although this belief may seem self-evident at first glance, contemporary education theories and data from a limited number of studies in medical education provide little affirmation for this concept, [20,21] and the goals of these earlier clinical encounters may vary. Better academic and clinical performances are certainly among the most important goals.

SNUCM also designed a clinical clerkship schedule to facilitate interactions between medical students and patients. However, the wide variation in the number of patient encounters during clerkship showed that clinical clerkship is not yet standardized, and we can assume that there is some qualitative variation in clinical clerkships as well as quantitative ones. In our study, we were astonished by data showing that the minimum number of patient encounters was zero in certain clerkships. This could be explained by students' under reporting their experiences with patients. Previous studies reported variable accuracy in logbooks, which usually involved significant under-reporting but little over reporting [22-24]. That is to say, students do not seem to overestimate exposure to patients or core problems, but they may not report every exposure. In addition, some clerkships 
emphasize observation and training in special skills rather than patient encounters, and in some clerkships, such as obstetrics and gynecology, access to patients is limited due to the privacy of patients, while most other clerkships set tasks for encountering patients and keeping medical records. Thus, a fair number of students do not have adequate clinical exposure to problems and lack the opportunity to acquire the skills necessary for competency as a physician [25-28].

In this study, students thought that the clinical clerkship was helpful for enhancing OSCE scores, but also felt that role playing or reading books dealing with OSCE were more helpful than their clinical clerkship. Previous studies reported that medical students evaluated exposure to real patients as being very important [10], while performance on the OSCE examination was related to well-organized study methods, not to clinical experience [7]. Another study reported that prior academic performance, rather than preparatory studying time, is a better predictor of OSCE outcomes [29]. Our results also show that GPA was correlated with OSCE scores. Clinical performance is very complex in nature and requires various training and teaching methods, and a certain level of knowledge is crucial for appropriate clinical performance.

Our study suggests that clinical experience during clerkship is weakly associated with student's clinical performance. Although only small correlations were present between them, the amount of clinical experience cannot be overlooked. We analyzed only the amount of clinical experience, without considering the variety of experiences or the quality of the supervision. Teaching quality and a supportive house staff were regarded as important elements for improving student performance during clerkships $[10,30]$. All of these should be considered when designing a clinical clerkship program.

We also focused on the differences between the number of patients for whom a medical history was taken and physical examinations were performed. Students do not always do a physical examination on every patient they encounter. The explanation for this could be any of the following: students may be hesitant to do a physical examination as they are not confident in their skills to conduct the physical examination, and are afraid of asking patients to take off' their clothes before doing the physical examination. Additionally, many physicians do not do physical examination thoroughly, and the importance of the physical examination has declined with the development of diagnostic aids. Therefore, students may have little chance of observing physicians doing physical examinations during clerkship.

Our study has several limitations. First, the OSCE examination, especially those using checklist rating systems, could be insufficient for accurately evaluating the clinical competency of students. Moreover, we tested only 8 cases, which may not be a sufficient number to evaluate clinical competency. Second, the number of patients that students encountered during clerkship could be inaccurate. The students recorded the number of patients for whom they took a medical history and performed a physical examination based on the logbook, but the accuracy of students' completion of the logbook was not systematically examined. Bias could be introduced in the recording of information. Finally, our study analyzed the correlation between the amount of clinical experience and the clinical competency of the student. We did not consider the qualitative aspects of the clinical experiences. Qualitative evaluations of clinical experiences as well as quantitative ones could have a significant correlation with the clinical performance scores of students.

\section{Conclusions}

In conclusion, there was small but positive relationship between clinical experience and students' performance on the OSCE. Increasing the amount of clinical experience as well as the quality of this experience should be re-enforced during clinical clerkship.

\section{Ethical approval}

This study was approved by the Institutional Review Board of Seoul National University College of Medicine. (IRB No. C-1202-040-397).

\section{Additional files}

Additional file 1: The format of the scoring rubric of OSCE.

Additional file 2: Questionnaire for student.

Competing interests

The authors declare that they have no competing interests.

\section{Authors' contributions}

SJM served as principal investigator and was responsible for the research design, ethics approval and authorship of the manuscript. JYK assisted in all steps of the project and was a major contributor of data collection and analysis. Both authors read and approved the final manuscript.

Received: 11 June 2014 Accepted: 29 September 2014 Published: 2 October 2014

\section{References}

1. Snell LM, Battles JB, Bedford JA, Washington ET: Verifying the curriculum of a family medicine clerkship. Med Educ 1998, 32(4):370-375.

2. Witzke DB, Koff NA, McGeagh AM, Skinner PD: Developing a computerbased system to document and report students' clinical patient encounters. Acad Med 1990, 65(7):440-441.

3. Kossoff EH, Hubbard TW, Gowen CW Jr: Early clinical experience enhances third-year pediatrics clerkship performance. Acad Med 1999, 74(11):1238-1241.

4. Ottenheijm RP, Zwietering PJ, Scherpbie AJ, Metsemakers JF: Early studentpatient contacts in general practice: an approach based on educational principles. Med Teach 2008, 30(8):802-808

5. Diemers AD, Dolmans $\mathrm{DH}$, Verwijnen MG, Heineman $\mathrm{E}$, Scherpbier AJ: Students' opinions about the effects of preclinical patient contacts on their learning. Adv Health Sci Educ Theory Pract 2008, 13(5):633-647. 
6. Wenrich MD, Jackson MB, Wolfhagen I, Ramsey PG, Scherpbier AJ: What are the benefits of early patient contact?-A comparison of three preclinical patient contact settings. BMC Med Educ 2013, 13:80

7. Martin IG, Stark P, Jolly B: Benefiting from clinical experience: the influence of learning style and clinical experience on performance in an undergraduate objective structured clinical examination. Med Educ 2000 34(7):530-534

8. Van Leeuwen YD, Dusman H, Mol SS, Pollemans MC, Drop MJ, Grol RP, Van Der Vleuten CP: Factors influencing the growth in knowledge of trainees in general practice. Adv Health Sci Educ Theory Pract 1997, 2(1):61-70.

9. McManus IC, Richards P, Winder BC, Sproston KA: Clinical experience, performance in final examinations, and learning style in medical students: prospective study. BMJ 1998, 316(7128):345-350.

10. Dolmans DH, Wolfhagen $I H$, Essed GG, Scherpbier AJ, Van der Vleuten CP. The impacts of supervision, patient mix, and numbers of students on the effectiveness of clinical rotations. Acad Med 2002, 77(4):332-335.

11. Wimmers PF, Schmidt HG, Splinter TA: Influence of clerkship experiences on clinical competence. Med Educ 2006, 40(5):450-458

12. Farnan JM, Petty LA, Georgitis E, Martin S, Chiu E, Prochaska M, Arora VM: A systematic review: the effect of clinical supervision on patient and residency education outcomes. Acad Med 2012, 87(4):428-442.

13. Rudaz A, Gut AM, Louis-Simonet M, Perrier A, Vu NV, Nendaz MR: Acquisition of clinical competence: added value of clerkship real-life contextual experience. Med Teach 2013, 35(2):e957-e962.

14. Harden RM, Gleeson FA: Assessment of clinical competence using an objective structured clinical examination (OSCE). Med Educ 1979, 13(1):41-54.

15. Harden RM, Stevenson M, Downie WW, Wilson GM: Assessment of clinical competence using objective structured examination. Br Med J 1975, 1(5955):447-451.

16. Jefferies A, Simmons B, Tabak D, Mcllroy JH, Lee KS, Roukema H, Skidmore M: Using an objective structured clinical examination (OSCE) to assess multiple physician competencies in postgraduate training. Med Teach 2007, 29(2-3):183-191.

17. Sudan R, Lynch TG, Risucci DA, Blair PG, Sachdeva AK: American College of Surgeons Resident Objective Structured Clinical Examination: a national program to assess clinical readiness of entering postgraduate year 1 surgery residents. Ann Surg 2014, 260(1):65-71.

18. Changiz T, Jamshidian S, Entezari MH, Kassaian N: Training and validation of standardized patients for evaluation of general practitioners' performance in management of obesity and overweight. Adv Biomed Res 2014, 3:77.

19. Shirazi M, Labaf A, Monjazebi F, Jalili M, Mirzazadeh M, Ponzer S, Masiello I: Assessing medical students' communication skills by the use of standardized patients: emphasizing standardized patients' quality assurance. Acad Psychiatry 2014, 38(3):354-360

20. Jolly BC, Jones A, Dacre JE, Elzubeir M, Kopelman P, Hitman G: Relationships between students' clinical experiences in introductory clinical courses and their performances on an objective structured clinical examination (OSCE). Acad Med 1996, 71(8):909-916.

21. Chatenay M, Maguire T, Skakun E, Chang G, Cook D, Warnock GL: Does volume of clinical experience affect performance of clinical clerks on surgery exit examinations? Am J Surg 1996, 172(4):366-372.

22. Denton GD, DeMott C, Pangaro LN, Hemmer PA: Narrative review: use of student-generated logbooks in undergraduate medical education. Teach Learn Med 2006, 18(2):153-164.

23. Denton GD, Hoang T, Prince L, Moores L, Durning S: Accuracy of medical student electronic logbook problem list entry. Teach Learn Med 2007, 19(4):347-351.

24. Ferenchick G, Mohmand A, Mireles J, Solomon D: Using patient encounte logs for mandated clinical encounters in an internal medicine clerkship. Teach Learn Med 2009, 21(4):299-304.

25. Fincher RM, Lewis LA: Learning, experience, and self-assessment of competence of third-year medical students in performing bedside procedures. Acad Med 1994, 69(4):291-295.

26. Taylor DM: Undergraduate procedural skills training in Victoria: is it adequate? Med J Aust 1997, 166(5):251-254.

27. Benson J, Quince T, Hibble A, Fanshawe T, Emery J: Impact on patients of expanded, general practice based, student teaching: observational and qualitative study. BMJ 2005, 331(7508):89.
28. Mol SS, Peelen JH, Kuyvenhoven MM: Patients' views on student participation in general practice consultations: a comprehensive review. Med Teach 2011, 33(7):e397-e400

29. Mavis BE: Does studying for an objective structured clinical examination make a difference? Med Educ 2000, 34(10):808-812.

30. Griffith CH III, Wilson JF, Haist SA, Ramsbottom-Lucier M: Do students who work with better housestaff in their medicine clerkships learn more? Acad Med 1998, 73(10 Suppl):S57-S59.

doi:10.1186/1472-6920-14-209

Cite this article as: Kim and Myung: Could clinical experience during clerkship enhance students' clinical performance? BMC Medical Education 2014 14:209.

\section{Submit your next manuscript to BioMed Central and take full advantage of:}

- Convenient online submission

- Thorough peer review

- No space constraints or color figure charges

- Immediate publication on acceptance

- Inclusion in PubMed, CAS, Scopus and Google Scholar

- Research which is freely available for redistribution 